\title{
Research Article \\ Cooperative Guidance of Seeker-Less Missiles for Precise Hit
}

\author{
Jianbo Zhao $\mathbb{1}$ and Fenfen Xiong $\mathbb{1}$ \\ School of Aerospace Engineering, Beijing Institute of Technology, Beijing 100081, China \\ Correspondence should be addressed to Jianbo Zhao; zhjb_cn@126.com
}

Received 18 October 2018; Revised 13 February 2019; Accepted 6 March 2019; Published 16 April 2019

Guest Editor: Manuel Soler

Copyright (c) 2019 Jianbo Zhao and Fenfen Xiong. This is an open access article distributed under the Creative Commons Attribution License, which permits unrestricted use, distribution, and reproduction in any medium, provided the original work is properly cited.

\begin{abstract}
A novel cooperative guidance scenario is proposed that implements fire-and-forget attacks for seeker-less missiles with a cheap finder for stationary targets and without requiring real-time communication among missiles or precise position information. Within the proposed cooperative scenario, the classic leader-follower framework is utilized, and a two-stage cooperative guidance law is derived for the seeker-less missile. Linear-quadratic optimal control and biased proportional navigation guidance (PNG) are employed to develop this two-stage cooperative guidance law to minimize the control cost in the first stage and to reduce the maximum acceleration command in the second stage when the acceleration command is continuous. Simulations and comparisons are conducted that demonstrate the effectiveness and advantages of the proposed guidance law.
\end{abstract}

\section{Introduction}

Homing guidance systems that can implement fire-andforget attacks have been rapidly developed and widely applied $[1,2]$. In recent years, studies on homing guidance have dealt with constraints in the angle of impact [3], mostly through the employment of optimal control theory [4-13]. Moreover, constraints on the maximum acceleration command are also commonly considered [14, 15]. As is wellknown, a seeker is essential to homing guidance systems, which is, however, very expensive. Therefore, it has become necessary to study guidance systems for seeker-less missiles.

Studies on the guidance of seeker-less missiles are mainly classified into two categories. The first category employs external guidance [1], whereby a ground station is utilized to control the trajectory of the seeker-less missile and to implement a precise hit $[16,17]$. However, due to the involvement of a ground station, the fire-and-forget aspect of the missile attack cannot be implemented, and the position of the ground station is likely to be exposed. The other category is a scenario in which cooperative guidance is utilized, in which the seeker-less missile is guided by a separate missile with a seeker to hit the target $[18,19]$. These two aforementioned cooperative guidance methods require realtime communication among the missiles and information on their precise position. It is well-known that the position of seeker-less missiles is commonly measured by the INS/GNSS (Inertial Navigation System and Global Navigation Satellite System), which inevitably induces localization errors. Moreover, real-time communication among missiles makes radio-silent attacks impossible, and the content of communications may be received and decoded by opponents, inducing difficulty for the defense penetration of missiles.

To address the aforementioned issues, a new cooperative guidance scenario is proposed to implement fire-and-forget attacks for seeker-less missiles without requiring real-time communication and precise position information. In this scenario, an onboard finder that is much cheaper than a seeker is employed for a seeker-less missile. Even though the measurement information produced by such a finder (the line-of-sight angle) is identical to that of the seeker, the finder has the following features that can clearly reduce costs, as the object of measurement would be another missile in the missile cluster: (1) the lock-on distance is remarkably reduced and (2) the requirements for identification capabilities are reduced, as information on the precise design of other missiles can be derived in advance, and there is no active disturbance or invisibility among these missiles. Therefore, this finder may be considered a cheaper version of a seeker, with a reduced lock-on distance and reduced requirements for 
identification capabilities. To differentiate this device from commonly used seekers, this device will be defined in this paper as a "finder." Due to its features, a seeker-less missile with this finder will not be able to employ certain common laws of guidance, for a reduced lock-on distance and reduced identification capabilities for the finder would result in the target being undetectable.

Within this proposed cooperative guidance scenario, a missile with a seeker that can independently hit the target is located in the front of the missile cluster during the flight, while other seeker-less missiles equipped with finders can hit the target in sequence by tracking the position of the nearest missile at the front, including the missile with the seeker. In this scenario, the employment of the finder reduces costs, and the number of cooperative missiles may be conveniently increased, considering the reduced lock-on distance. In addition, to implement the aforementioned cooperative guidance scenario, a two-stage guidance law is designed for the seekerless missile by employing linear-quadratic optimal control and biased proportional navigation guidance (PNG).

The remainder of this paper is organized as follows. In Section 2, the novel cooperative guidance scenario is introduced. In Section 3, the two-stage guidance law employing linear-quadratic optimal control and biased PNG is described for the implementation of the cooperative guidance scenario. In Section 4, the simulation results are presented and analyzed to verify the effectiveness and superiority of the proposed guidance law. Conclusions are drawn in the last section.

\section{The Proposed Cooperative Guidance Scenario}

Within the proposed cooperative guidance scenario (as shown in Figure 1), the classic leader-follower framework is employed, where $n$ seeker-less missiles with finders are guided by a missile with a seeker (denoted as $L$ ) to cooperatively hit a stationary target. Considering the relatively short lock-on distance of the finder, the number of cooperative missiles, i.e., $n$, can be conveniently increased in this proposed cooperative guidance scenario. In Figure 1, only missile $L$ is equipped with a seeker, and the seeker-less missile can easily implement a fire-and-forget attack by tracking the position of the nearest missile in front using its finder. In this work, for any two missiles with a tracking relationship, the tracked missile is considered as the leader and the tracking one the follower. Therefore, as shown in Figure 1, missile $L$ is the leader of the missiles $F_{1}, F_{2}, \ldots, F_{n}$, and missile $F_{1}$ is the leader of the missiles $F_{11}, F_{12}, \ldots, F_{1 n}$, etc.

In this cooperative guidance scenario, the missile $L$ with the seeker could directly employ certain common guidance laws (e.g., PNG) to achieve an accurate hit, while the seeker-less missile can merely track its leader according to the information on relative motion derived by the finder. Considering that the finder of the follower missile will be ineffective if the lead missile has already hit its target, the law of guidance for seeker-less missiles is designed to be separated into two stages based on whether the leader has hit the target. Clearly, for the second stage of the seeker-less missile (i.e., its leader has hit the target), the initial relative motion between the target and seeker-less missile is derived by the

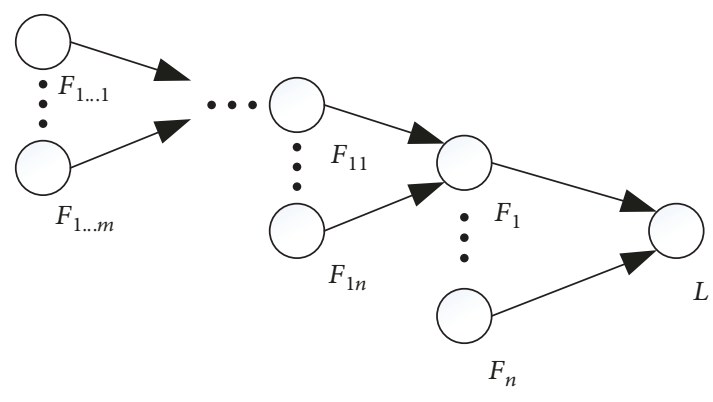

FIGURE 1: Proposed cooperative guidance scenario.

finder with the assumption that the leader will finally arrive at the stationary target; thus, the seeker-less missile can implement certain common guidance laws (e.g., PNG) as the rate of change in the line-of-sight (LOS) angle for the target is derived in real time via the relative kinematic equations. Therefore, a precise hit by the seeker-less missile is achievable at the end of the second stage of guidance. Clearly, cooperative guidance between the seeker-less missile and its leader only exists during the first stage of guidance.

For the two-stage law of guidance for the seeker-less missile to be effective, any impact between the seeker-less missile and its leader must be avoided in the first stage, which will be studied later (Remark 2 of Section 3.2). In addition, during the first stage of guidance, the total cost of control for the seeker-less missile is minimized to save energy (as is commonly done in practice), while for the second stage of guidance, the maximum acceleration command is minimized in order to reduce requirements on the actuator.

\section{Guidance Law Formulation}

3.1. Model Linearization. Considering that the proposed cooperative guidance scenario consists of multiple groups with a leader-follower framework, a single leader-follower framework is first illustrated. During the first stage of a seeker-less missile (as a leader), its followers can be considered to be in the middle guidance stage, which is out of the scope of this work and thus not introduced here. Therefore, the missile $L$ or only seeker-less missiles in the second stage are considered as leaders. The geometry between the leader $M_{l}$, follower $M_{i}$, and the target in the inertial reference coordinate $X-O-Y$ is shown in Figure 2, in which the variables with the subscripts $i$ and $l$, respectively, represent the states of the follower and leader in motion. Moreover, $V, a, \theta, q$, and $r$ denote the speed, normal acceleration, heading angle, LOS angle, and LOS distance, respectively.

The speed of all missiles is considered to be constant in this work, and the relative kinematic equations between the leader and follower are given as follows:

$$
\begin{aligned}
\dot{r}_{i} & =-V_{i} \cos \left(q_{i}-\theta_{i}\right)+V_{l} \cos \left(q_{i}-\theta_{l}\right), \\
r_{i} \dot{q}_{i} & =V_{i} \sin \left(q_{i}-\theta_{i}\right)-V_{l} \sin \left(q_{i}-\theta_{l}\right), \\
\eta_{i} & =\theta_{i}-q_{i},
\end{aligned}
$$




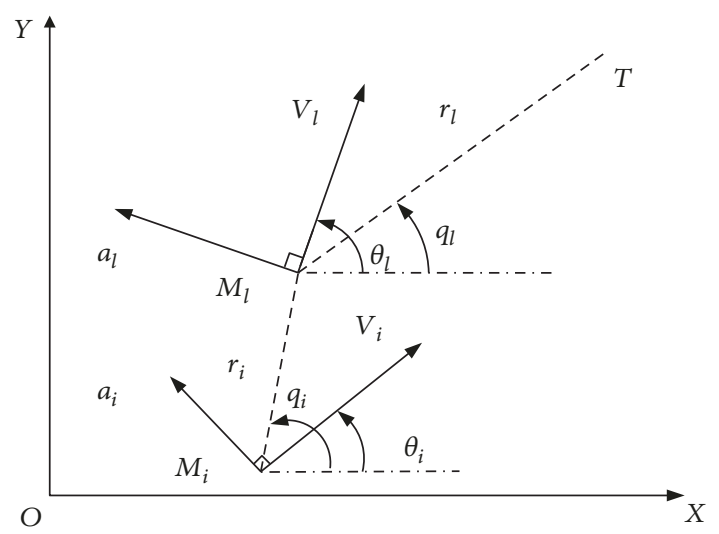

FIGURE 2: Geometry among the leader, follower, and target.

where $\eta$ denotes the lead angle. The normal acceleration command of the follower is given as

$$
a_{i}=V_{i} \dot{\theta}_{i}
$$

We define the error between the position coordinate for the leader and follower relative to the $y$-axis as $y_{i}$; thus,

$$
\dot{y}_{i}=V_{l} \sin \theta_{l}-V_{i} \sin \theta_{i} .
$$

To simplify the derivation of the guidance law, a linearization process is implemented. We approximate that there is no normal acceleration for the leader, as will be illustrated in Section 3.3. We also assume that $\theta_{i}$ is sufficiently small [20]. The reason is that for the leader implements the rectilinear motion and the $x$-axis of the employed inertial reference coordinate is defined as being approximately along the motion direction of the leader; the heading angle of the leader is small. Moreover, the heading angle of the follower would be essentially identical to that of the leader, as the terminal guidance for a maneuvering target commonly ends up in a tail-chase situation and the tracking target for the follower is the leader in this work. Therefore, the heading angle of the follower $\left(\theta_{i}\right)$ can be regarded as sufficiently small. The derivative of equation (5) is taken to be

$$
\ddot{y}_{i}=-a_{i} .
$$

As the leader flies straight, it is assumed without loss of generality that $\theta_{l}=0$. The state vector is defined as

$$
X=\left[y_{i}, \dot{y}_{i}, \theta_{i}\right]
$$

Therefore, the linearized guidance model can be derived as

$$
\dot{X}=A X+B u,
$$

where

$$
\begin{aligned}
& A=\left[\begin{array}{lll}
0 & 1 & 0 \\
0 & 0 & 0 \\
0 & 0 & 0
\end{array}\right], \\
& B=\left[\begin{array}{c}
0 \\
-1 \\
1 / V_{i}
\end{array}\right], \\
& u=a_{i} .
\end{aligned}
$$

3.2. Optimal Control Problem. As the follower only requires the employment of the common guidance law in the second stage, it is assumed that the PNG is utilized without loss of generality. Therefore, for the first stage of guidance, the following cost function is introduced to reduce the maximum acceleration for the second stage (the first term) and to minimize the control effort (the second term).

$$
J=\frac{w}{2} \eta_{i}^{2}\left(t_{\mathrm{If}}\right)+\frac{b}{2} \int_{t_{0}}^{t_{\mathrm{f}}} u^{2}(t) d t
$$

where $b$ is a positive constant and $t_{0}$ and $t_{\mathrm{fl}}$ denote the status at the initial and terminal instant of time, respectively, for the first stage of guidance. The constant $w$ is defined as

$$
w=\frac{c N_{i}^{2} V_{i}^{4}}{r_{i}^{2}\left(t_{\mathrm{lf}}\right)}
$$

where $c$ is a positive constant and $N_{i}$ is the navigation gain of follower $M_{i}$ during the second stage of guidance. An explanation for the choice of $w$ will be provided as the 2nd point of Remark 2.

As $q_{i}$ is assumed to be sufficiently small, it can be approximated as

$$
q_{i}=\frac{y_{i}}{r_{i}}
$$

Therefore, according to equation (3), the lead angle of the follower $\eta_{i}$ can be formulated as

$$
\eta_{i}=\theta_{i}-\frac{y_{i}}{r_{i}}
$$

Substituting equation (13) into equation (10) yields

$$
J=\left.\frac{w}{2}\left(\theta_{i}-\frac{y_{i}}{r_{i}}\right)^{2}\right|_{t=t_{\mathrm{fl}}}+\frac{b}{2} \int_{t_{0}}^{t_{\mathrm{fl}}} u^{2}(t) d t .
$$

Then, the cost function shown in equation (14) can be classically reformulated as

$$
J=\frac{1}{2} X^{T}\left(t_{\mathrm{lf}}\right) P\left(t_{\mathrm{lf}}\right) X\left(t_{\mathrm{lf}}\right)+\frac{1}{2} \int_{t_{0}}^{t_{\mathrm{fl}}} u^{T}(t) b u(t) d t,
$$


where

$$
\begin{gathered}
P=\left[\begin{array}{cc}
{\left[P_{11}\right]_{1 \times 1}} & {\left[P_{12}\right]_{1 \times 2}} \\
{\left[P_{12}{ }^{T}\right]_{2 \times 1}} & {\left[P_{22}\right]_{2 \times 2}}
\end{array}\right], \\
P_{11}=w / r_{i}^{2}, P_{12}=\left[0-w / r_{i}\right], P_{22}=\left[\begin{array}{ll}
0 & 0 \\
0 & w
\end{array}\right] .
\end{gathered}
$$

It is well-known that the solution of the optimal control problem formulated by equations (8) and (15) is

$$
u=-\frac{B^{T} K(t) X(t)}{b},
$$

where $K(t)$ is derived by numerically solving the associated differential matrix Riccati equation formulated as

$$
\begin{aligned}
\dot{K} & =-K A-A^{T} K+\frac{K B B^{T} K}{b}, \\
K\left(t_{\mathrm{fl}}\right) & =P .
\end{aligned}
$$

The matrixes $A, B$, and $K(t)$ can be reformulated as

$$
\begin{aligned}
A & =\left[\begin{array}{cc}
{[0]_{1 \times 1}} & {\left[A_{12}\right]_{1 \times 2}} \\
{[0]_{2 \times 1}} & {[0]_{2 \times 2}}
\end{array}\right], \quad A_{12}=\left[\begin{array}{ll}
1 & 0
\end{array}\right], \\
B & =\left[\begin{array}{c}
{[0]_{1 \times 1}} \\
{\left[B_{21}\right]_{2 \times 1}}
\end{array}\right], \quad B_{21}=\left[\begin{array}{c}
-1 \\
\frac{1}{V_{i}}
\end{array}\right], \\
K & =\left[\begin{array}{cc}
{\left[K_{11}\right]_{1 \times 1}} & {\left[K_{12}\right]_{1 \times 2}} \\
{\left[K_{12}^{T}\right]_{2 \times 1}} & {\left[K_{22}\right]_{2 \times 2}}
\end{array}\right] .
\end{aligned}
$$

Substituting equations (19), (20), and (21) into equation (18) yields

$$
\begin{aligned}
& \dot{K}_{11}=K_{12} S K_{12}{ }^{T}, \quad K_{11}\left(t_{\mathrm{lf}}\right)=P_{11}, \\
& \dot{K}_{12}=-K_{11} A_{12}+K_{12} S K_{22}, \quad K_{12}\left(t_{\mathrm{lf}}\right)=P_{12}, \\
& \dot{K}_{22}=-K_{12}{ }^{T} A_{12}-A_{12}{ }^{T} K_{12}+K_{22} S K_{22}, \quad K_{22}\left(t_{\mathrm{lf}}\right)=P_{22},
\end{aligned}
$$

where $S$ is defined as $B_{21} B_{21}^{T} / b$.

Therefore, equation (17) can be rewritten as

$$
u=-\frac{B_{21}^{T}\left[K_{12}^{T}\left(t, t_{\mathrm{lf}}\right), K_{22}\left(t, t_{\mathrm{lf}}\right)\right] X(t)}{b} .
$$

Remark 1. $r_{i}\left(t_{\mathrm{lf}}\right)$ must be predetermined to calculate $P\left(t_{\mathrm{lf}}\right)$. Assuming that $q_{i}$ and $\theta_{i}$ are sufficiently small and that $\theta_{l}=$ 0 , it can be approximated that the follower flies along the straight-line trajectory of the leader. Therefore, $r_{i}\left(t_{\mathrm{lf}}\right)$ can be estimated by

$$
r_{i}\left(t_{\mathrm{lf}}\right)=r_{i}\left(t_{0}\right)-\left(V_{i}-V_{l}\right)\left(t_{\mathrm{fl}}-t_{0}\right),
$$

where $r_{i}\left(t_{0}\right)$ is derived by the finder at the initial time.

If the leader is the missile $L$, and by employing PNG, $t_{\mathrm{fl}}$ can be directly derived by the existing approach as [21]

$$
t_{\mathrm{fl}}=\frac{r_{l}\left(t_{0}\right)}{V_{l}}\left(1+\frac{\eta_{l}^{2}\left(t_{0}\right)}{2\left(2 N_{l}-1\right)}\right)+t_{0}
$$

where $N_{l}$ is the navigation gain of missile $L$. Moreover, the initial distance between the missile $L$ and the target $r_{L}\left(t_{0}\right)$ can be derived [3]. If the leader is a seeker-less missile (e.g., the missile $\left.F_{1}\right), t_{\mathrm{fl}}$ can be approximately derived as

$$
t_{\mathrm{fl}}=\frac{r_{L}\left(t_{0}\right)+r_{F_{1}}\left(t_{0}\right)}{V_{F_{1}}},
$$

where $V_{F_{1}}$ is the velocity of missile $F_{1}$ and $r_{F_{1}}\left(t_{0}\right)$ is the initial distance between the missile $L$ and $F_{1}$. Similarly, if the leader is the seeker-less missile $F_{11}$, equation (26) can be rephrased as

$$
t_{\mathrm{fl}}=\frac{\left(r_{L}\left(t_{0}\right)+r_{F_{1}}\left(t_{0}\right)\right)+r_{F_{11}}\left(t_{0}\right)}{V_{F_{11}}},
$$

where $V_{F_{11}}$ and $r_{F_{11}}\left(t_{0}\right)$ are the velocity of missile $F_{11}$ and the initial distance between missiles $F_{1}$ and $F_{11}$, respectively. Therefore, a follower is required to derive the distance between its leader and the target at the initial time.

Remark 2. The three criteria for the guidance mentioned in Section 2 can be satisfied.

(1) For the first stage of guidance, it can be derived from equation (24) that $\forall t \in\left[t_{0}, t_{\mathrm{ff}}\right]$,

$$
\begin{aligned}
& r_{i}(t) \geq r_{i}\left(t_{0}\right)-\left(V_{i}-V_{l}\right)\left(t_{\mathrm{fl}}-t_{0}\right), \quad \text { with } V_{i} \geq V_{l}, \\
& r_{i}(t) \geq r_{i}\left(t_{0}\right), \quad \text { with } V_{i}<V_{l} .
\end{aligned}
$$

Evidently, the impact between the leader and follower is avoided with the condition $V_{i}<V_{l}$. Moreover, when $V_{i} \geq V_{l}$, an impact can also be avoided if $r_{i}\left(t_{0}\right)$ satisfies

$$
r_{i}\left(t_{0}\right) \geq\left(V_{i}-V_{l}\right)\left(t_{\mathrm{fl}}-t_{0}\right) .
$$

In addition, by considering the limited lock-on distance of the finder, the upper limit of $r_{i}\left(t_{0}\right)$ which will be implemented during the middle guidance stage, can be derived, i.e., 


$$
\begin{aligned}
& r_{i}\left(t_{0}\right) \leq l_{d}, \quad \text { with } V_{i} \geq V_{l}, \\
& r_{i}\left(t_{0}\right) \leq l_{d}-\left(V_{l}-V_{i}\right)\left(t_{\mathrm{fl}}-t_{0}\right), \quad \text { with } V_{i}<V_{l},
\end{aligned}
$$

where $l_{d}$ denotes the lock-on distance of the finder.

(2) Because the follower can employ the PNG during the second stage of guidance, i.e.,

$$
a_{i}=N_{i} V_{i} \dot{q}_{i}
$$

Substituting equation (2) into equation (31) yields

$$
a_{i}=\frac{N_{i} V_{i}\left[V_{i} \sin \left(q_{i}-\theta_{i}\right)-V_{l} \sin \left(q_{i}-\theta_{l}\right)\right]}{r_{i}} .
$$

Since $V_{l}=0$ in the second stage, equation (32) can be rephrased as

$$
a_{i}=-\frac{N_{i} V_{i}^{2} \sin \eta_{i}}{r_{i}}
$$

As the initial acceleration of the PNG is at a maximum, the term $(w / 2) \eta_{i}^{2}\left(t_{\mathrm{fl}}\right)$ employed in equation (10) can reduce the maximum acceleration for the second stage $\left(\left|a_{i}\left(t_{\mathrm{fl}}\right)\right|\right)$.

(3) The total control cost for the first stage can be optimized by considering the term $(b / 2) \int_{t_{0}}^{t_{\mathrm{fl}}} u^{2}(t) d t$ in equation (10).

3.3. Two-Stage Guidance. Clearly, it is more convenient to implement the guidance algorithm if it is reformulated with respect to variables that can be directly measured by onboard instruments. Therefore, an alternative measurable state vector for the acceleration command during the first stage is provided in this subsection. With the assumptions that $\theta_{l}=$ 0 and $\theta_{i}$ is sufficiently small, equation (5) can be rephrased as

$$
\dot{y}_{i}=-V_{i} \theta_{i}
$$

Moreover, because equation (12) is identical to

$$
y_{i}=r_{i} q_{i}
$$

the state vector can be redefined as

$$
Z=\left[\theta_{i}, q_{i}\right]^{T},
$$

and it can be derived that

$$
X=W Z \text {, }
$$

where

$$
W=\left[\begin{array}{cc}
0 & r_{i} \\
-V_{i} & 0 \\
1 & 0
\end{array}\right]
$$

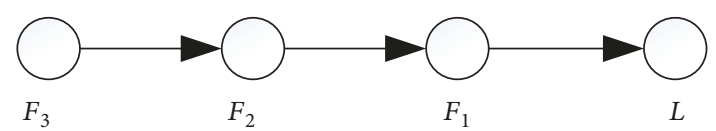

FIGURE 3: The relationship between the missiles.

TABLE 1: Initial states of the missiles.

\begin{tabular}{lcccc}
\hline Parameters & $L$ & $F_{1}$ & $F_{2}$ & $F_{3}$ \\
\hline Position, $\times 10^{3} \mathrm{~m}$ & $(6,1)$ & $(5,0.5)$ & $(4,0)$ & $(3,0.5)$ \\
Velocity, $\times 10^{2} \mathrm{~m} / \mathrm{s}$ & 3.5 & 3.7 & 3.7 & 3.7 \\
Heading angle $(\mathrm{deg})$ & 10 & 0 & 0 & 0 \\
\hline
\end{tabular}

Then, substituting equation (37) into equation (23) yields

$$
u=-\frac{B_{21}{ }^{T}\left[K_{21}{ }^{T}\left(t, t_{\mathrm{fl}}\right), K_{22}\left(t, t_{\mathrm{fl}}\right)\right] W Z(t)}{b} .
$$

In the second stage of guidance, if the follower employs the PNG, the acceleration command would be discontinuous at the initial time, since

$$
a_{i}\left(t_{\mathrm{lf}}^{-}\right) \neq N_{i} V_{i} \dot{q}_{i}\left(t_{\mathrm{lf}}{ }^{+}\right)
$$

where $t_{\mathrm{lf}}{ }^{+}$and $t_{\mathrm{lf}}{ }^{-}$represent the initial time of the second stage and the terminal time of the first stage, respectively. Therefore, a biased PNG is proposed for the seeker-less missile during the second stage, i.e.,

$$
a_{i}=N_{i} V_{i}\left(\dot{q}_{i}(t)-\dot{q}_{i}\left(t_{\mathrm{lf}}^{+}\right) h\right)+a_{i}\left(t_{\mathrm{lf}}^{-}\right) h,
$$

where

$$
h=\frac{r_{i}(t)}{r_{i}\left(t_{\mathrm{lf}}\right)} .
$$

Both $\eta_{i}\left(t_{\mathrm{lf}}{ }^{+}\right)$and $a_{i}\left(t_{\mathrm{lf}}^{-}\right)$are sufficiently small if optimal control is implemented in the first stage of guidance. Thus, $\left|\eta_{i}\right|<(\pi / 2)$ can be guaranteed during the second stage of guidance. Thus, both $r_{i}$ and $h$ are monotonously decreasing during this stage, which further means that equation (41) will converge to the PNG, and the miss distance as well as the terminal acceleration command of the biased PNG could be identical to 0 . Moreover, it is verified that the normal acceleration of the leader is approximately identical to 0 when the missile $L$ and the seeker-less missile in the second stage employ the PNG and the biased PNG, respectively, with greater navigation gain.

\section{Simulation and Analysis}

In our analysis, three seeker-less missiles with finders, guided by a missile with a seeker, cooperatively hit a stationary target located at $12000 \mathrm{~m}$ and $1000 \mathrm{~m}$. The measurement relationship between the missiles and their initial states of motion are presented in Figure 3 and Table 1, in which $L$ represents the missile with the seeker, while $F_{1}, F_{2}$, and $F_{3}$ represent seeker-less missiles. The missile $L$ employs the PNG, while 


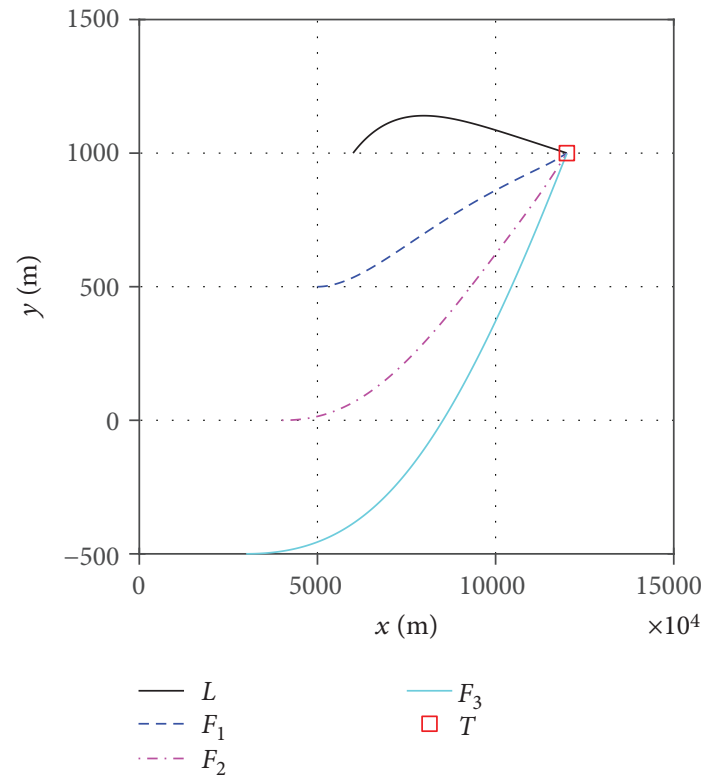

(a) Trajectory

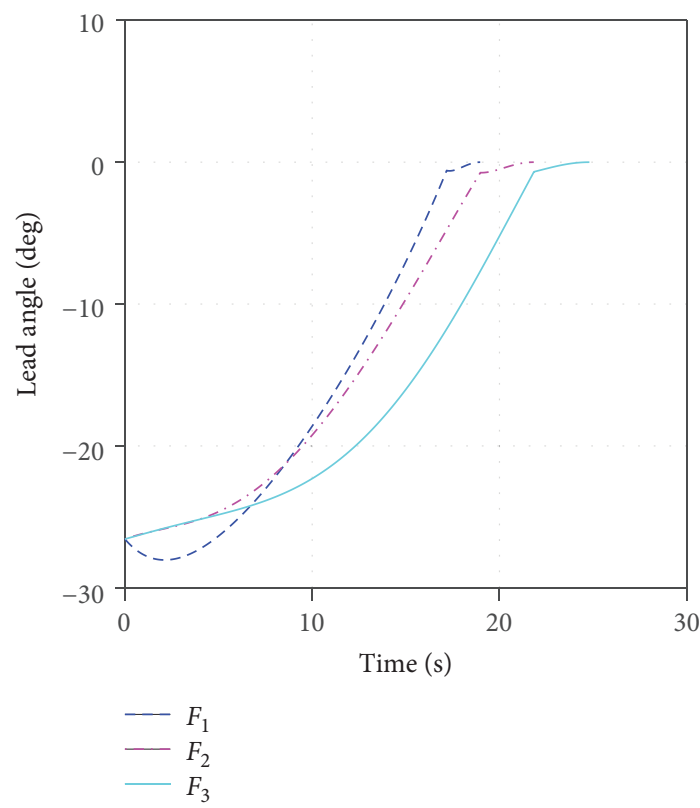

(c) Lead angle

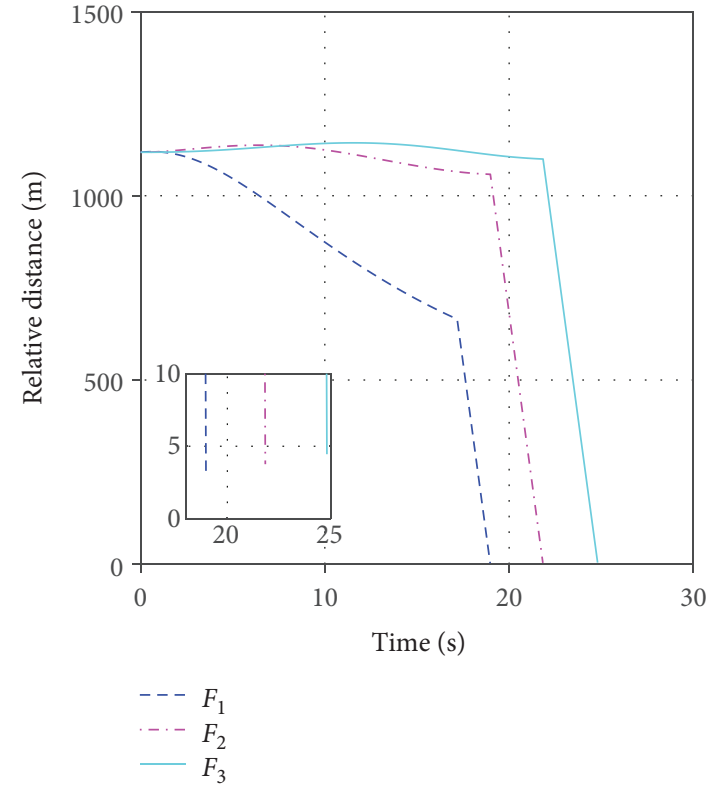

(b) Leader-follower distance

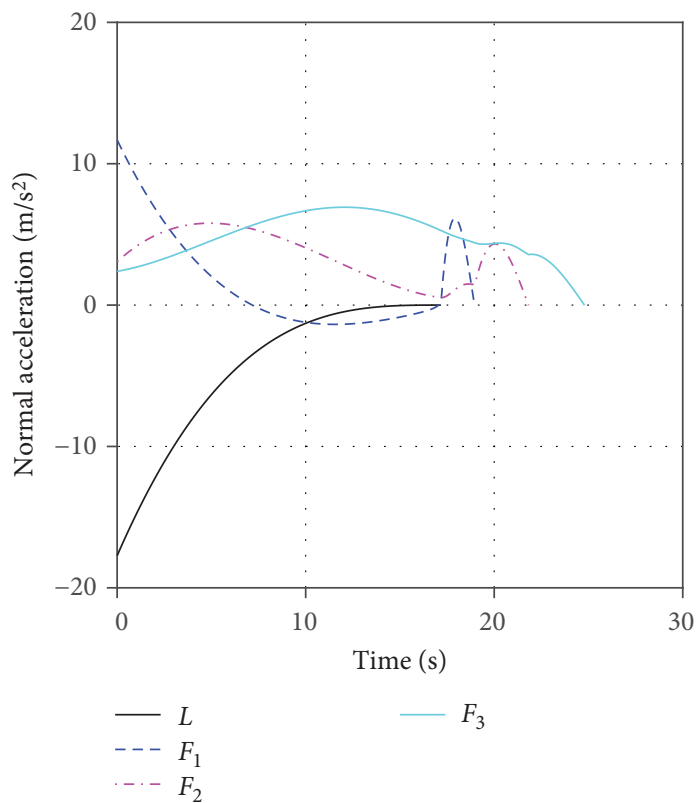

(d) Normal acceleration

Figure 4: Simulation results for the proposed cooperative guidance law.

$F_{1}, F_{2}$, and $F_{3}$ all employ the proposed or modified (for comparison) two-stage guidance law during the entire flight. These two-stage guidance laws change to second stage if the leader is lost for follower. In Figure 3, two different cases are considered: (1) the leader is a missile with a seeker (for $\left.F_{1}\right)$ and $(2)$ the leaders are seeker-less missiles with finders (for $F_{2}$ and $F_{3}$ ). To achieve a precise hit, the miss distance and normal acceleration command of these missiles and the relative distance between the leader and follower are required to be less than $5 \mathrm{~m}, 20 \mathrm{~m} / \mathrm{s}^{2}$, and $1200 \mathrm{~m} / \mathrm{s}^{2}$, respectively.

4.1. Sample Simulation. The simulation results for the proposed two-stage guidance law are presented in Figure 4.
The trajectories of the four missiles are illustrated in Figure 4(a), which indicates that all seeker-less missiles that correspond to the two cases could precisely hit their targets when the two-stage guidance law is employed for follower missiles with the leader in the first stage. Figure 4(b) shows the leader-follower distance (missile-target distance during second guidance stage), in which it indicates that all the seeker-less missiles can satisfy requirements for miss distances and lock-on distances of finders with the proposed cooperative guidance law. Figure 4(c) illustrates that the lead angles between the seeker-less missiles and their leaders are essentially identical to 0 at the terminal time instant for the first stage of guidance. In other words, the maximum normal 


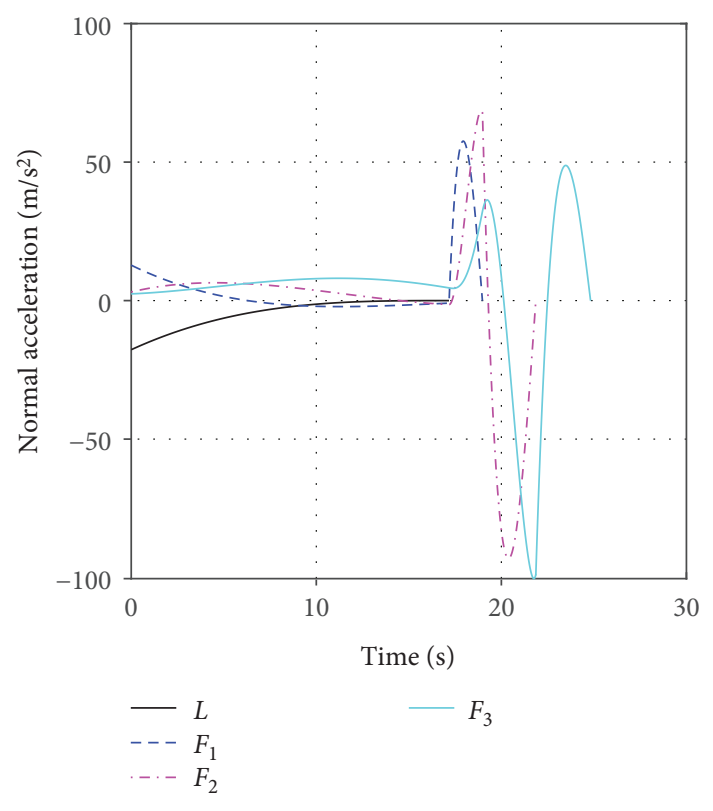

(a) 1st modified two-stage guidance law

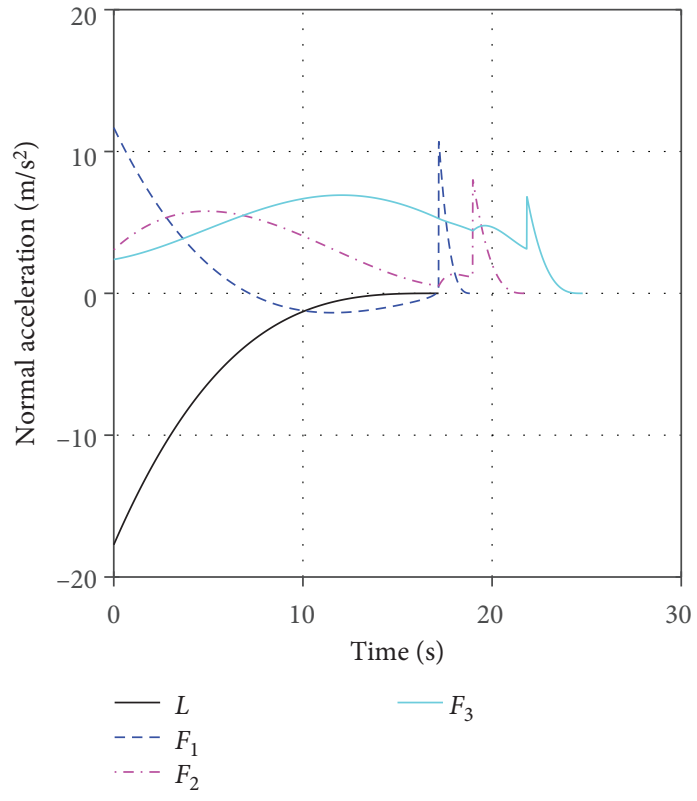

(b) 2nd modified two-stage guidance law

FIgURE 5: Normal acceleration command for modified guidance laws.

acceleration of the seeker-less missile for the second stage of guidance can be reduced. In Figure 4(d), it is indicated that all the missiles can satisfy requirements for a normal acceleration command. Moreover, from Figures 4(b) and 4(d), it can be verified that the miss distance as well as the terminal acceleration command of the seeker-less missile could be identical to 0 .

4.2. Comparison of Two Modified Two-Stage Guidance Laws. To verify the superiority of the proposed cost function for the first stage and the biased PNG for the second stage, two modified two-stage guidance laws using the existing formulations are introduced. For the first modified guidance law, the zeroeffort miss distance is considered to produce an optimal state of initial motion for the second stage of guidance, as is commonly done in the literature; i.e., the cost function shown in equation (10) is rewritten as [22]

$$
J=\frac{\varsigma}{2}\left[y_{i}\left(t_{\mathrm{fl}}\right)+\dot{y}_{i}\left(t_{\mathrm{fl}}\right) \cdot \Delta t\right]^{2}+\frac{\zeta}{2} \int_{t_{0}}^{t_{\mathrm{f}}} u^{2}(t) d t
$$

where $\varsigma$ and $\zeta$ are positive constants and $\Delta t$ denotes the error in flight time between the leader and follower, which is essentially identical to $\left(r_{i}\left(t_{0}\right)\right) / V$. For the second modified guidance law, the existing PNG is utilized for the second guidance stage instead of the proposed biased PNG.

With the first or second modified two-stage guidance law, all the miss distances of $F_{1}, F_{2}$, and $F_{3}$ and the relative distances between leaders and followers would satisfy the requirement, which can be verified by the simulation. However, compared with the results shown in Figure 4(d) for the proposed method, Figure 5(a), derived by the existing method, indicates that for the first modified two-stage guidance law, the maximum normal acceleration of the seeker- less missile during the second stage of guidance is far greater and cannot satisfy the requirement. The reason is that, compared with the cost function introduced in equation (10), equation (43) cannot effectively reduce the maximum normal acceleration during the second stage of guidance. Moreover, by comparing Figures 4(d) and 5(b), it is observed that compared with the PNG, the seeker-less missile employing the proposed biased PNG can clearly obtain a continuous acceleration command and a smaller maximum acceleration during the second stage of guidance.

\section{Conclusion}

To achieve a precise hit of a seeker-less missile for a stationary target, a novel cooperative scenario for guidance is proposed in this work that is able to implement fire-and-forget attacks for seeker-less missiles without real-time communication or precise position information. Within the proposed novel cooperative guidance scenario, a two-stage cooperative guidance law is derived for a seeker-less missile. The guidance law for the first stage can produce the minimum control cost for this stage as well as the reduced maximum acceleration command for the second stage. The guidance law for the second stage can guarantee a precise hit for a seeker-less missile with a continuous acceleration command. Simulation results illustrate the effectiveness and superiority of the proposed two-stage guidance law.

\section{Data Availability}

The data used to support the findings of this study are included within the article. 


\section{Conflicts of Interest}

The authors declare that there is no conflict of interest regarding the publication of this paper.

\section{Acknowledgments}

This work was supported by the National Science Foundation of China (grant number 11532002) and the Hongjian Innovation Foundation of China (grant number BQ203HYJJ-Q2018002).

\section{References}

[1] G. M. Siouris, Missile Guidance and Control Systems, SpringerVerlag New York, Inc, 2004.

[2] J. ZHAO and S. YANG, "Integrated cooperative guidance framework and cooperative guidance law for multi-missile," Chinese Journal of Aeronautics, vol. 31, no. 3, pp. 546-555, 2018.

[3] N. Balhance, M. Weiss, and T. Shima, "Cooperative guidance law for intrasalvo tracking," Journal of Guidance Control and Dynamics, vol. 40, no. 6, pp. 1441-1456, 2017.

[4] R. Livermore and T. Shima, "Deviated pure-pursuit-based optimal guidance law for imposing intercept time and angle," Journal of Guidance, Control, and Dynamics, vol. 41, no. 8, pp. 1807-1814, 2018.

[5] S. F. Xiong, M. Y. Wei, M. Y. Zhao, H. Xiong, W. H. Wang, and B. C. Zhou, "Hyperbolic tangent function weighted optimal intercept angle guidance law," Aerospace Science and Technology, vol. 78, pp. 604-619, 2018.

[6] H. Q. Zhang, S. J. Tang, J. Guo, and W. Zhang, "A two-phased guidance law for impact angle control with seeker's field-ofview limit," International Journal of Aerospace Engineering, vol. 2018, Article ID 7403639, 13 pages, 2018.

[7] J. H. Liu, J. Y. Shan, and Q. Liu, "Optimal pulsed guidance law with terminal impact angle constraint," Proceedings of the Institution of Mechanical Engineers, Part G: Journal of Aerospace Engineering, vol. 231, no. 11, pp. 1993-2005, 2017.

[8] K. S. Erer and R. Tekin, "Impact time and angle control based on constrained optimal solutions," Journal of Guidance Control and Dynamics, vol. 39, no. 10, pp. 2448-2454, 2016.

[9] B. G. Park, T. H. Kim, and M. J. Tahk, "Range-to-go weighted optimal guidance with impact angle constraint and seeker's look angle limits," IEEE Transactions on Aerospace and Electronic Systems, vol. 52, no. 3, pp. 1241-1256, 2016.

[10] R. Tsalik and T. Shima, "Optimal guidance around circular trajectories for impact-angle interception," Journal of Guidance, Control, and Dynamics, vol. 39, no. 6, pp. 1278-1291, 2016.

[11] C. H. Lee, J. I. Lee, and M. J. Tahk, "Sinusoidal function weighted optimal guidance laws," Proceedings of the Institution of Mechanical Engineers, Part G: Journal of Aerospace Engineering, vol. 229, no. 3, pp. 534-542, 2015.

[12] Y. Zhao, J. B. Chen, and Y. Z. Sheng, "Terminal impact angle constrained guidance laws using state-dependent Riccati equation approach," Proceedings of the Institution of Mechanical Engineers, Part G: Journal of Aerospace Engineering, vol. 229, no. 9, pp. 1616-1630, 2015.

[13] A. Maity, H. B. Oza, and R. Padhi, "Generalized model predictive static programming and angle-constrained guidance of air-to-ground missiles," Journal of Guidance, Control, and Dynamics, vol. 37, no. 6, pp. 1897-1913, 2014.

[14] C. H. Lee, T. H. Kim, M. J. Tahk, and I. H. Whang, "Polynomial guidance laws considering terminal impact angle and acceleration constraints," IEEE Transactions on Aerospace and Electronic Systems, vol. 49, no. 1, pp. 74-92, 2013.

[15] R. Tekin and K. S. Erer, "Switched-gain guidance for impact angle control under physical constraints," Journal of Guidance, Control, and Dynamics, vol. 38, no. 2, pp. 205-216, 2015.

[16] G. S. Kumar, R. Ghosh, D. Ghose, and A. Vengadarajan, "Guidance of seekerless interceptors using innovation covariance based tuning of Kalman filters," Journal of Guidance, Control, and Dynamics, vol. 40, no. 3, pp. 603-614, 2017.

[17] G. S. Kumar, D. Ghose, and A. Vengadarajan, "An integrated estimation/guidance approach for seeker-less interceptors," Proceedings of the Institution of Mechanical Engineers, Part G: Journal of Aerospace Engineering, vol. 229, no. 5, pp. 891$905,2015$.

[18] Q. L. Zhao, J. Chen, X. W. Dong, Q. D. Li, and Z. Ren, “Cooperative guidance law for heterogeneous missiles intercepting hypersonic weapon," Acta Aeronautica et Astronautica Sinica, vol. 37, no. 3, pp. 936-948, 2016.

[19] E. Zhao, S. Wang, T. Chao, and M. Yang, "Multiple missiles cooperative guidance based on leader-follower strategy," Proceedings of 2014 IEEE Chinese Guidance, Navigation and Control Conference, 2014, pp. 1163-1167, Yantai, China, 2014.

[20] C. K. Ryoo, H. J. Cho, and M. J. Tahk, “Optimal guidance laws with terminal impact angle constraint," Journal of Guidance, Control, and Dynamics, vol. 28, no. 4, pp. 724-732, 2005.

[21] I. S. Jeon, J. I. Lee, and M. J. Tahk, "Homing guidance law for cooperative attack of multiple missiles," Journal of Guidance, Control, and Dynamics, vol. 33, no. 1, pp. 275-280, 2010.

[22] P. Zarchan, Tactical and Strategic Missile Guidance, VA: AIAA, 6th edition, 2012. 


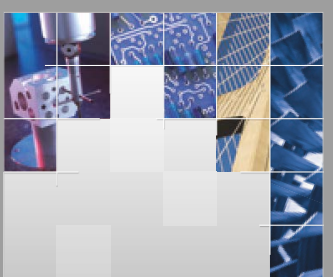

\section{Enfincering}
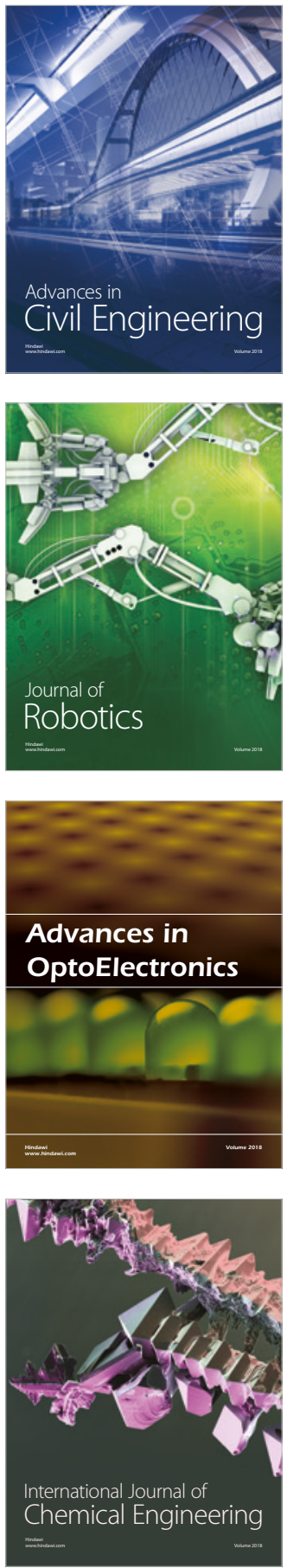

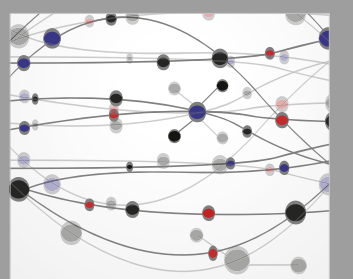

\section{Rotating \\ Machinery}

The Scientific World Journal

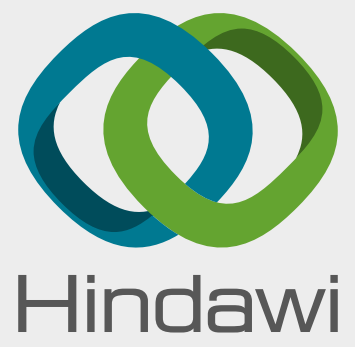

Submit your manuscripts at

www.hindawi.com
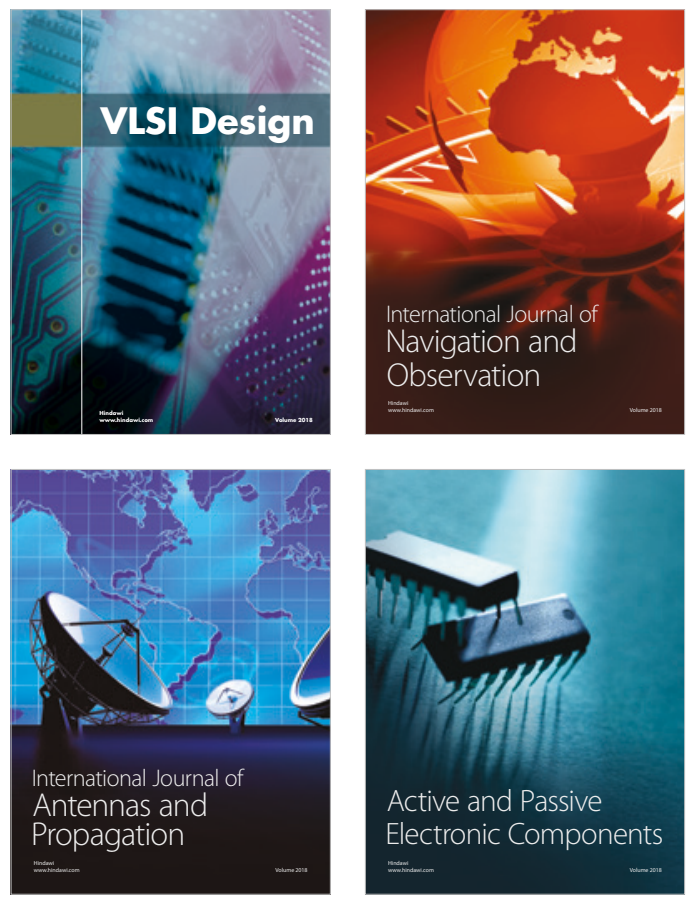
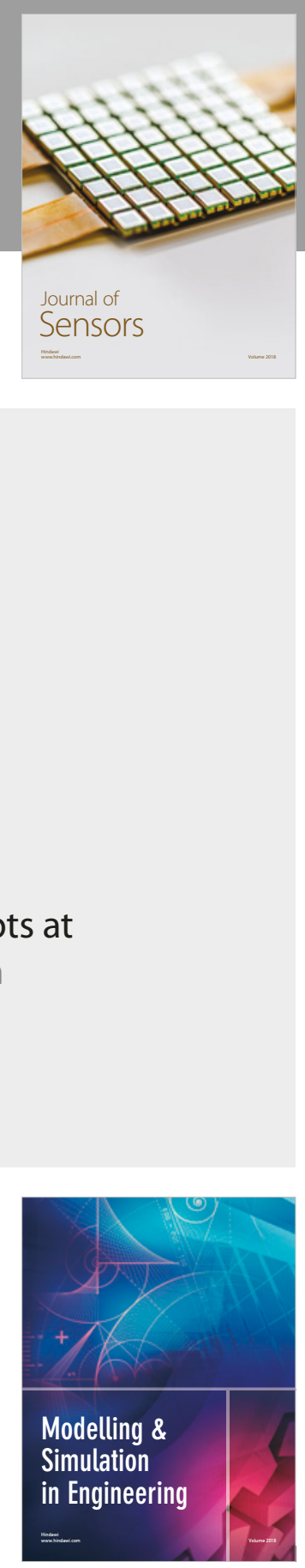

\section{Advances \\ Multimedia}
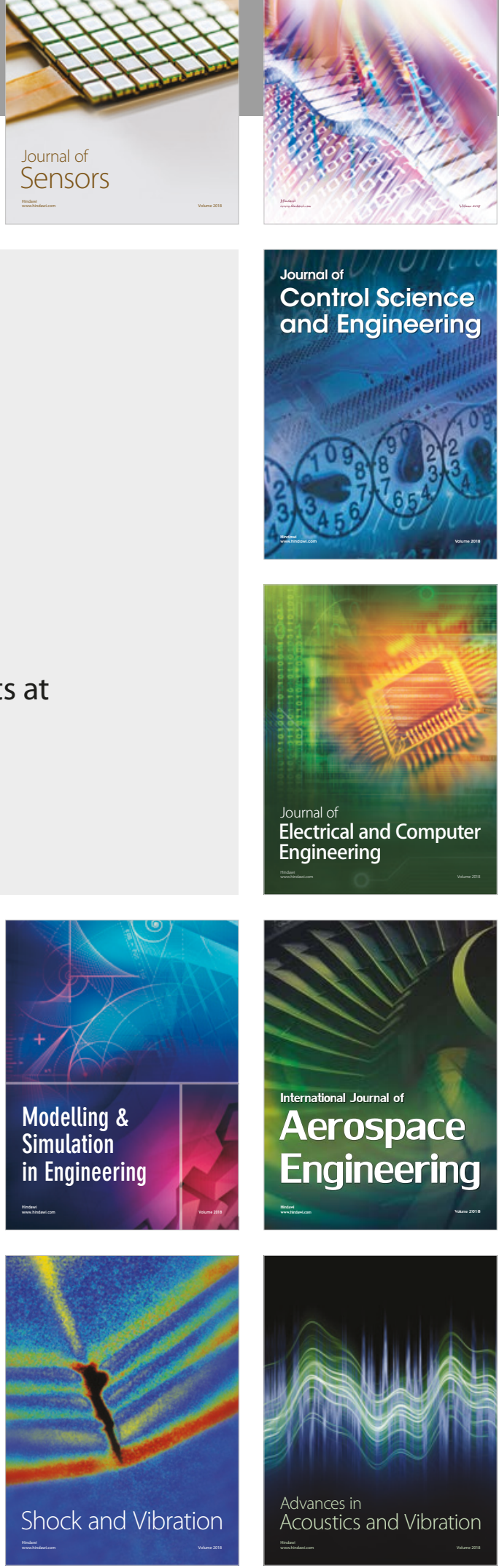